\title{
Transcatheter arterial chemoembolization and radiation therapy for treatment-naïve patients with locally advanced hepatocellular carcinoma
}

\author{
Sang Won Kim, MD*, Dongryul Oh, MD²*, Hee Chul Park, MD, PhD², Do Hoon Lim, MD, PhD², \\ Sung Wook Shin, MD, PhD ${ }^{3}$, Sung Ki Cho, MD, PhD², Geum-Youn Gwak, MD, PhD ${ }^{4}$, \\ Moon Seok Choi, MD, PhD, Yong Han Paik, MD, PhD ${ }^{4}$, Seung Woon Paik, MD, PhD ${ }^{4}$ \\ 'Department of Radiation Oncology, Yeungnam University Medical Center, Daegu; Departments of ${ }^{2}$ Radiation Oncology, \\ ${ }^{3}$ Radiology, and ${ }^{4}$ Medicine, Samsung Medical Center, Sungkyunkwan University School of Medicine, Seoul, Korea
}

Purpose: To evaluate the safety and efficacy of transcatheter arterial chemoembolization (TACE) followed by radiotherapy (RT) in treatment-naïve patients with locally advanced hepatocellular carcinoma (HCC).

Materials and Methods: Eligibility criteria were as follows: newly diagnosed with $\mathrm{HCC}$, the Barcelona Clinic Liver Cancer stage C, Child-Pugh class A or B, and no prior treatment for HCC. Patients with extrahepatic spread were excluded. A total of 59 patients were retrospectively enrolled. All patients were treated with TACE followed by RT. The time interval between TACE and RT was 2 weeks as per protocol. A median RT dose was $47.25 \mathrm{~Gy}_{10}$ as the biologically effective dose using the $\alpha / \beta=10$ (range, 39 to 65.25 Gy $\left.y_{10}\right)$.

Results: At 1 month, complete response was obtained in 3 patients (5\%), partial response in 27 patients (46\%), stable disease in 13 patients (22\%), and progressive disease in 16 patients (27\%). The actuarial one- and two-year OS rates were $60.1 \%$ and $47.2 \%$, respectively. The median OS was 17 months (95\% confidence interval, 5.6 to 28.4 months). The median time to progression was 4 months (range, 1 to 35 months). Grade 3 or greater liver enzyme elevation occurred in only two patients (3\%) after RT. Grade 3 gastroduodenal toxicity developed in two patients (3\%).

Conclusion: The combination treatment of TACE followed by RT with two-week interval was safe and it showed favorable outcomes in treatment-naïve patients with locally advanced HCC. A prospective randomized trial is needed to validate these results.

Keywords: Hepatocellular carcinoma, Transcatheter arterial chemoembolization, Radiotherapy

\section{Introduction}

The Barcelona Clinic Liver Cancer (BCLC) staging system provides a guide for the management of hepatocellular carcinoma (HCC) that is endorsed by both the European
Association for the Study of the Liver and the American Association for the Study of Liver Diseases [1,2]. The BCLC stage $\mathrm{C}$ refers to advanced $\mathrm{HCC}$, and it is defined as an Eastern Cooperative Oncology Group (ECOG) performance status of 1 or 2; evidence of vascular invasion or extrahepatic disease;

Received 31 December 2013, Revised 6 March 2014, Accepted 13 March 2014.

Correspondence: Hee Chul Park, MD, PhD, Department of Radiation Oncology, Samsung Medical Center, Sungkyunkwan University School of Medicine, 81 Irwon-ro, Gangnam-gu, Seoul 135-710, Korea. Tel: +82-2-3410-2605, Fax: +82-2-3410-2619, E-mail: rophc@skku.edu

*These authors contributed equally to the article.

(c) This is an Open Access article distributed under the terms of the Creative Commons Attribution Non-Commercial License (http://creativecommons.org/ licenses/by-nc/3.0/) which permits unrestricted non-commercial use, distribution, and reproduction in any medium, provided the original work is properly cited.

www.e-roj.org 
or both. Sorafenib is the current standard treatment for BCLC stage $C$ based on evidence from randomized clinical trials $[3,4]$. In addition to sorafenib, many locoregional modalities, such as transcatheter arterial chemoembolization (TACE), hepatic arterial infusion chemotherapy (HAIC), radiation therapy (RT), and different combinations of these treatments, have been used to treat advanced HCC in Asian countries [5]. These locoregional modalities provide maximal reduction in primary tumor burden for as long as the liver function and/or performance status of individual patients permits. These treatments may provide a survival benefit because more than two-thirds of patients with advanced HCC die of hepatic failure or intrahepatic tumor progression rather than metastatic disease [6]. One of the locoregional treatment strategies is the combination of TACE and RT. Several reports demonstrated the synergic effect of the combination of TACE and RT [7-12]. Since 2008, based on these rationales, our institution has treated advanced HCC with TACE followed 2 weeks later by RT as a protocol [13].

Although several institutions have reported favorable outcomes with this combined treatment approach [7-9,1418], interpreting the results of these studies is controversial due to heterogeneity in study populations, histories of prior treatments, and the strategies used to combine TACE and RT. The purpose of this study was to evaluate the efficacy of combined TACE followed 2 weeks later by RT among treatment-naïve patients with BCLC stage $C$ of which prognosis was validated in several studies $[19,20]$ to limit potential confounding effects by prior treatments for HCC and heterogeneity of study population.

\section{Materials and Methods}

\section{Patients}

Between March 2008 and December 2010, a total of 204 patients were treated by combined TACE followed by RT for locally advanced HCC. Among them, patients who met the following criteria were enrolled in the current study: 1) newly diagnosed with HCC, 2) BCLC stage $C$, 3) Child-Pugh class $A$ or $B$, and 4) no prior treatment for HCC (treatment-naïve). Patients with extrahepatic spread were excluded. Finally, 59 patients were analyzed retrospectively.

\section{Treatment protocol}

The details of treatment protocol were described in our previous report [13]. All patients were treated with one course of TACE followed by RT. The time interval between TACE and RT was 2 weeks as per protocol. All patients underwent preRT clinical evaluation by physical examination and blood tests. If levels of aspartate aminotransferase (AST), alanine aminotransferase (ALT), or both were elevated three-fold or more above normal levels and/or the Child-Pugh score was elevated by two or more, RT was delayed by one week. After the course of TACE and RT, we again evaluate the patient's general condition, liver function and tumor response. If complete remission was achieved, the patient was followedup regularly. Otherwise, the additional TACE was repeated to control the residual tumors as long as the liver function and/or performance status of individual patients permits.

\section{TACE procedure}

Selective arteriography of the hepatic artery was performed to locate the tumor and any tumor neovascularity. After identifying the tumor-feeding artery, a mixture of Adriamycin (Dong-A Pharm., Seoul, Korea) and Lipiodol (Guerbet, Aulnaysous-Bois, France) was slowly injected through the catheter. The Adriamycin and Lipiodol doses depended on the tumor size and vascularity ( $3 \mathrm{mg}$ of Adriamycin and $1 \mathrm{~mL}$ of Lipiodol per $1 \mathrm{~cm}$ diameter of tumor), although we limited the doses in a single TACE session to $70 \mathrm{mg}$ of Adriamycin and $25 \mathrm{~mL}$ of Lipiodol. When embolization with a Lipiodol mixture alone was insufficient to block tumor-feeding arteries, additional embolization with 1- to 2-mm diameter gelatin sponges (Cutanplast; Mascia Brunelli Spa, Milano, Italy) was performed. The end point of TACE was complete Lipiodol uptake within the tumor or stagnant blood flow in the tumor-feeding arteries by fluoroscopy or injection of the maximum Lipiodol dose.

\section{RT}

All patients underwent four-dimensional computed tomography (4D CT) simulation to consider tumor movement during breathing. Before simulation, patients were trained to take a regular and shallow breath while aided by a goggle display system, which showed their own respiratory waveform for each patient. On simulation, a CT scan (General Electric, Milwaukee, WI, USA) with contrast-enhancement was obtained for the arterial and portal phases during quiet breathing. Next the respiratory image sets aided by the prerecorded goggle display were acquired with a real-time position management system (Varian Medical Systems, Palo Alto, CA, USA). The respiratory phase was divided into ten equal phases with $0 \%$ as end-inspiration and 50\% as end-expiration (0\% to 90\%). 
The images of arterial and portal phases and four respiratory phases $(0 \%, 30 \%, 50 \%$, and $80 \%)$ were used for target delineation.

The gross tumor volumes (GTV), including the main tumors and/or portal vein tumor thrombosis (PVTT), were delineated at each phase. A 7-mm margin was added to the GTV within the liver to obtain the clinical target volume (CTV); non-enhancing thrombosis was also included within the CTV. The internal target volume (ITV) was determined from the sum of the CTV for each phase. A 5-mm margin was added to the ITV to create the PTV.

The total radiation dose was determined by tentative guideline in our institution $[13,21]$. It was determined according to the percentage of the normal liver volume irradiated by more than $50 \%$ of the prescribed dose $\left(V_{50 \% \text { dose }}\right)$ as follows: for a $V_{50 \% \text { dose }}$ below 20\%, a total dose of 50 Gy was given in 10 fractions; for a $V_{50 \% \text { dose }}$ between $20 \%$ and $35 \%$, a total dose of 45 Gy was given in 10 fractions; for a $V_{50 \% \text { dose }}$ between $35 \%$ and $50 \%$, a total dose of 40 Gy was given in 10 fractions; and for a $V_{50 \% \text { dose }}$ above 50\%, a total dose of 35 Gy was given in 10 fractions. When the gastroduodenal (GD) wall received a full dose of the prescribed dose, a total dose of $33 \mathrm{~Gy}$ in 11 fractions or 44 Gy in 22 fractions was prescribed to avoid GD toxicity. If the normal liver volume defined as the whole liver minus the CTV was less than $700 \mathrm{~mL}$, RT was not recommended because of the concern for the liver toxicity in patients with cirrhotic liver.

To verify the target position, we obtained gated onboard images (OBIs) of the exhalation phase daily before each treatment, and we matched these OBls to dynamic reconstruction and rendering (DRR) images that were reconstructed with 50\% phase CT images. The visualization of liver dome outline of 50\% phase on DRR images was used for easy matching. Target localization was performed with the liver dome or Lipiodol deposits as surrogates. In case of shifts greater than $5 \mathrm{~mm}$ in positions, the $\mathrm{OBI}$ was re-taken. When the position shift was still greater than $5 \mathrm{~mm}$, automatic adjustment for the patient's couch position was performed by the linear accelerator machine.

\section{Evaluation}

All patients were examined at least once a week during treatment. After the completion of RT, patients were followedup at 1 month. A triphasic CT scan was performed for response evaluation. The radiological response was assessed with the modified Response Evaluation Criteria in Solid Tumors. For patients of whom the GD wall was in close proximity to the
PTV, fiberoptic gastroduodenoscopy was performed before and after RT to evaluate GD toxicity. Any treatment-related adverse events were evaluated at each visit with the Common Terminology Criteria for Adverse Events ver. 3.0.

\section{Statistical analysis}

The duration of overall survival (OS) was calculated from the start date of TACE to the date of death or last follow-up. Time to progression was calculated from the start date of TACE to the date of any progression or last follow-up. Survival rates were estimated by the Kaplan-Meier method. Survival differences between groups were compared by the log-rank test. The Cox proportional hazards regression model was employed for multivariate analysis. The $p$-values of 0.05 or less (two-sided) were considered statistically significant SPSS ver. 19.0 (IBM, Armonk, NY, USA) was used for the analysis.

\section{Results}

\section{Patient characteristics}

Forty-nine patients (83\%) started RT at 2 weeks after the completion of TACE. RT was delayed 1 week in 10 patients (17\%), but all delays were due to patients' personal schedules and not impaired liver function. A median total dose of $35 \mathrm{~Gy}$ (range, 30 to $45 \mathrm{~Gy}$ ) was delivered in 2 to $4.5 \mathrm{~Gy}$ per fraction. The calculated biologically effective dose as the $\alpha / \beta=10$ ranged from 39 to $65.25 \mathrm{~Gy}_{10}$ (median $47.25 \mathrm{~Gy}_{10}$ ), and it was ranged from 32.5 to $54.4 \mathrm{~Gy}$ (median $39.4 \mathrm{~Gy}$ ) when converted to the $2-G y$ equivalent dose with $\alpha / \beta=10$. The median age was 55 years (range, 27 to 76 years). The characteristics of all patients are summarized in Table 1.

\section{Response}

The overall response to treatment was evaluated at 1 month after the completion of RT. Complete response (CR) was obtained in 3 patients (5\%), partial response (PR) in 27 patients (46\%), stable disease (SD) in 13 patients (22\%), and progressive disease (PD) in 16 patients (27\%). Of the 16 patients with PD, target lesion PD developed in 6 patients, non-target lesion PD developed in 4 patients, and new lesions developed in 14 patients. Tumor response within the irradiated field (RT-infield response) was also evaluated; CR was obtained in 3 patients (5\%), PR in 31 patients (53\%), SD in 23 patients (39\%), and PD in 2 patients (3\%). 
Table 1. Patient characteristics

\begin{tabular}{|c|c|}
\hline Characteristic & No. of patients $(\%)$ \\
\hline \multicolumn{2}{|l|}{ Gender } \\
\hline Male & $50(85)$ \\
\hline Female & $9(15)$ \\
\hline \multicolumn{2}{|l|}{ Age (yr) } \\
\hline$<55$ & $29(53)$ \\
\hline$\geq 55$ & $30(47)$ \\
\hline \multicolumn{2}{|l|}{ ECOG performance } \\
\hline 0 & $22(37)$ \\
\hline 1 & 37 (63) \\
\hline \multicolumn{2}{|l|}{ Child-Pugh class } \\
\hline A & $52(88)$ \\
\hline B & 7 (12) \\
\hline \multicolumn{2}{|l|}{ Viral marker } \\
\hline HBV & $50(85)$ \\
\hline $\mathrm{HCV}$ & $2(3)$ \\
\hline Both HBV and HCV & $2(3)$ \\
\hline Non-B and Non-C & $5(9)$ \\
\hline \multicolumn{2}{|l|}{ Modified UICC T stage } \\
\hline 2 & $3(5)$ \\
\hline 3 & $22(37)$ \\
\hline 4 & $34(58)$ \\
\hline Tumor size (cm), median (range) & $9(2-18)$ \\
\hline$\leq 5$ & $12(20)$ \\
\hline$>5$ and $\leq 10$ & $18(31)$ \\
\hline$>10$ & $29(49)$ \\
\hline \multicolumn{2}{|l|}{ Vascular invasion } \\
\hline No & $3(5)$ \\
\hline Yes & $56(95)$ \\
\hline Main PV & $33(56)$ \\
\hline Segmental PV & $21(36)$ \\
\hline HV or IVC & $6(10)$ \\
\hline \multicolumn{2}{|l|}{ Multiplicity } \\
\hline Single & $23(39)$ \\
\hline Multiple & $36(61)$ \\
\hline \multicolumn{2}{|l|}{ Initial AFP (ng/mL) } \\
\hline$<400$ & $23(39)$ \\
\hline$\geq 400$ & $36(61)$ \\
\hline
\end{tabular}

ECOG, Eastern Cooperative Oncology Group; HBV, hepatitis B; HCV, hepatitis C; UICC, Union for International Cancer Control; AFP, alpha-fetoprotein.

\section{Additional treatment after combined TACE and RT}

For intrahepatic tumor control, a median of 2 additional sessions (range, 1 to 8 sessions) of TACE was performed in 53 patients (88\%), and re-irradiation was performed in five patients (6\%). Sorafenib was used for 12 patients (21\%) for extrahepatic spread.

\section{Survival and failure}

The median follow-up duration for all patients was 10 months (range, 2 to 44 months). At the time of analysis, 32 patients (49\%) were still alive. Fig. 1 depicts the case history of one of the long-term survivors. The actuarial one- and two-year OS rates were $60.1 \%$ and $47.2 \%$, respectively. The median OS was 17 months (95\% confidence interval, 5.6 to 28.4) (Fig. 2). On univariate anlaysis for OS, Child-Pugh class A (A vs. B, $p=$ 0.011), modified Union for International Cancer Control T2-3 stage (T2-3 vs. T4, $p=0.020$ ), single tumor (single vs. multiple tumors, $p=0.006$ ), the absence of main PVTT (no vs. yes, $p=$ 0.005), and overall response to treatment ( $C R$ and PR vs. SD and $P D, p<0.001)$ were statistically significant factors for better OS (Fig. 3). Gender ( $p=0.841$ ), age ( $>55$ vs. $\leq 55$ years, $p$ $=0.504)$, performance status (ECOG 0 vs. $1, p=0.206$ ), tumor size ( $>10$ vs. $\leq 10 \mathrm{~cm}, p=0.088$ ), the initial level of alphafetoprotein (>400 vs. $\leq 400 \mathrm{ng} / \mathrm{mL}, p=0.718$ ) and RT dose ( $>47.5$ vs. $\leq 47.5 \mathrm{~Gy}_{10}, \mathrm{p}=0.749$ ) did not show any statistically significant differences. Multivariate analysis showed that Child-Pugh class $(p=0.002)$, the presence of main PVTT ( $p$ $=0.026)$, multiplicity $(p=0.022)$, and overall response to treatment ( $p=0.019$ ) were statistically significant prognostic factors for $0 \mathrm{~S}$.

During follow-up, a total of 59 patients (88\%) experienced disease progression. The median time to progression was 4 months (range, 1 to 35 months). The actuarial one- and twoyear progression-free survival rates were $20.3 \%$ and $8.5 \%$, respectively (Fig. 2). At the initial recurrence, intrahepatic metastasis (29 patients, 49\%) was the most frequent pattern of failure, followed by primary tumor failure (23 patients, 39\%) and extrahepatic metastasis (11 patients, 19\%).

\section{Adverse events}

Table 2 summarizes the data on acute toxicity during TACE and RT or 1 month after completion of RT. Grade 3 or greater liver enzyme elevation (i.e., more than five times the upper limit of the normal value) occurred in 41 patients (69\%) after TACE, but it was self-limiting in all patients, and no patients delayed the start of RT for that reason. Grade 3 or greater liver enzyme elevation occurred in only two patients (3\%) after RT. During follow-up, grade 2 GD toxicity developed in 14 patients (24\%) and grade 3 GD toxicity developed in two patients (3\%). No treatment-related deaths were observed. 

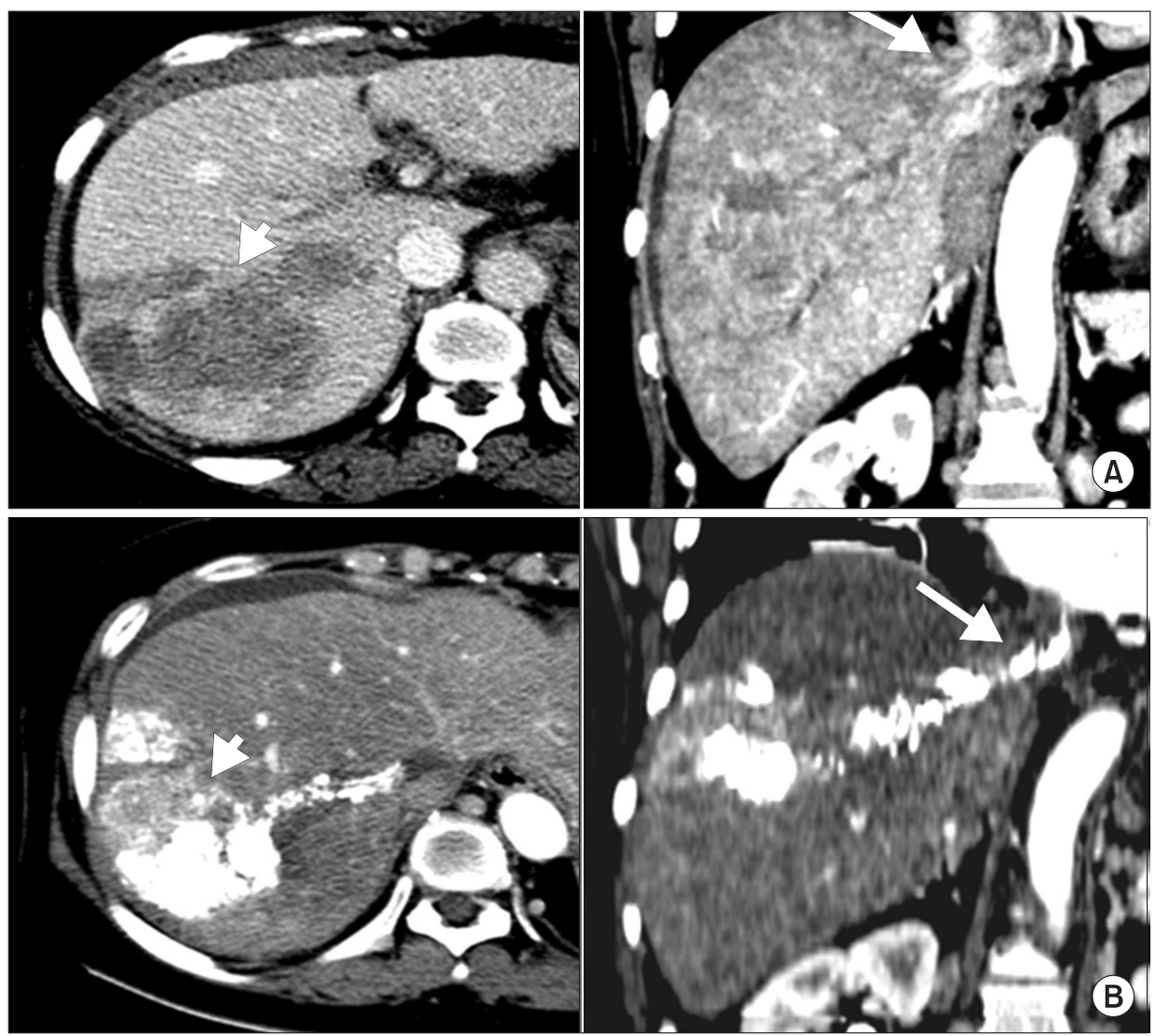

Fig. 1. Tumor response after transcatheter arterial chemoembolization (TACE) followed by radiotherapy (RT). A 42-year-old woman was diagnosed with hepatocellular carcinoma in dynamic computed tomography (CT). (A) The CT image showed an $8.5-\mathrm{cm}$ mass (short arrow) extending to the inferior vena cava (IVC) through the right hepatic vein (long arrow). The initial alpha-fetoprotein (AFP) level was $19,600 \mathrm{ng} / \mathrm{mL}$. The patient was treated with TACE and RT with $35 \mathrm{~Gy}$
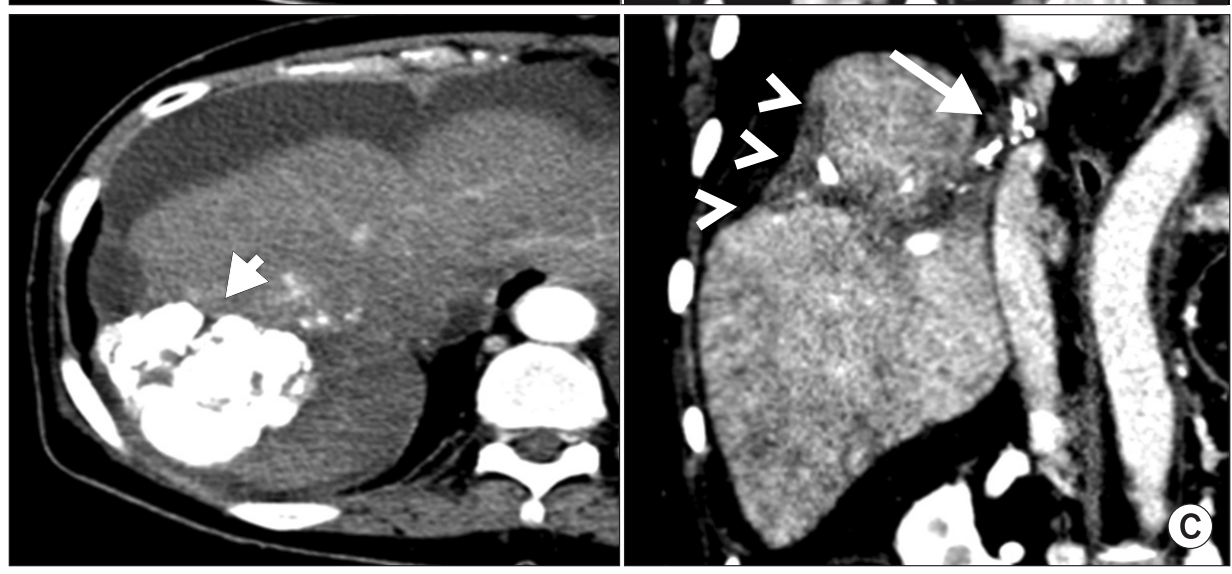
(3.5 Gy per fraction). (B) At 1 month, partial response was obtained. Partial lipiodol uptake was observed in the large mass extending to the IVC (long arrow), but viable tumors were still enhanced (short arrow) The AFP level decreased to 1,375 ng/ $\mathrm{mL}$, and two sessions of TACE were performed additionally. (C) At 35 months after treatment, there was no evidence of recurrence on $\mathrm{CT}$, and the AFP level was $3.8 \mathrm{ng} / \mathrm{mL}$. Liver atrophy was observed (arrowheads).

\section{Discussion and Conclusion}

The BCLC staging systems provides the treatment strategies based on the results of several clinical trials. Recently, the results of two large-scale randomized controlled trials in advanced-stage HCC indicate that sorafenib can improve median OS by nearly 3 months compared with placebo $[3,4]$ : median OS was improved from 7.9 to 10.7 months in the Western trial, and from 4.2 to 6.5 months in the Asian-Pacific trial. Sorafenib has become the standard treatment for BCLC stage $C$ based on these data. However, the improvement in survival with sorafenib is modest, and BCLC stage C encompasses a wide spectrum of disease, ranging from vascular invasion, which indicates 'locally advanced' disease; to distant metastasis, which indicates 'systemic disseminated' disease. Indeed, the treatment for these two categories seems to be quite different. In Asian countries, both sorafenib and locoregional modalities, such as TACE, HAIC, and RT, have been used for locally advanced HCC $[5,22,23]$. These locoregional modalities were expected to improve survival by preventing local progression while preserving liver function. Moreover, there is no evidence showing that sorafenib is better than 
these locoregional modalities.

Combining TACE with RT is one locoregional strategy for treatment of advanced HCC. This strategy has some advantages

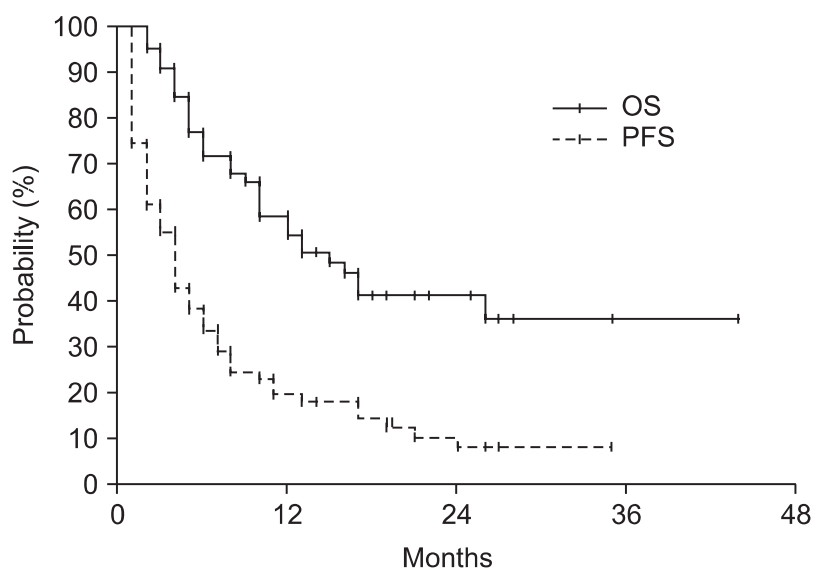

Fig. 2. Overall survival (OS) rate and progression-free survival (PFS) rate in all patients.
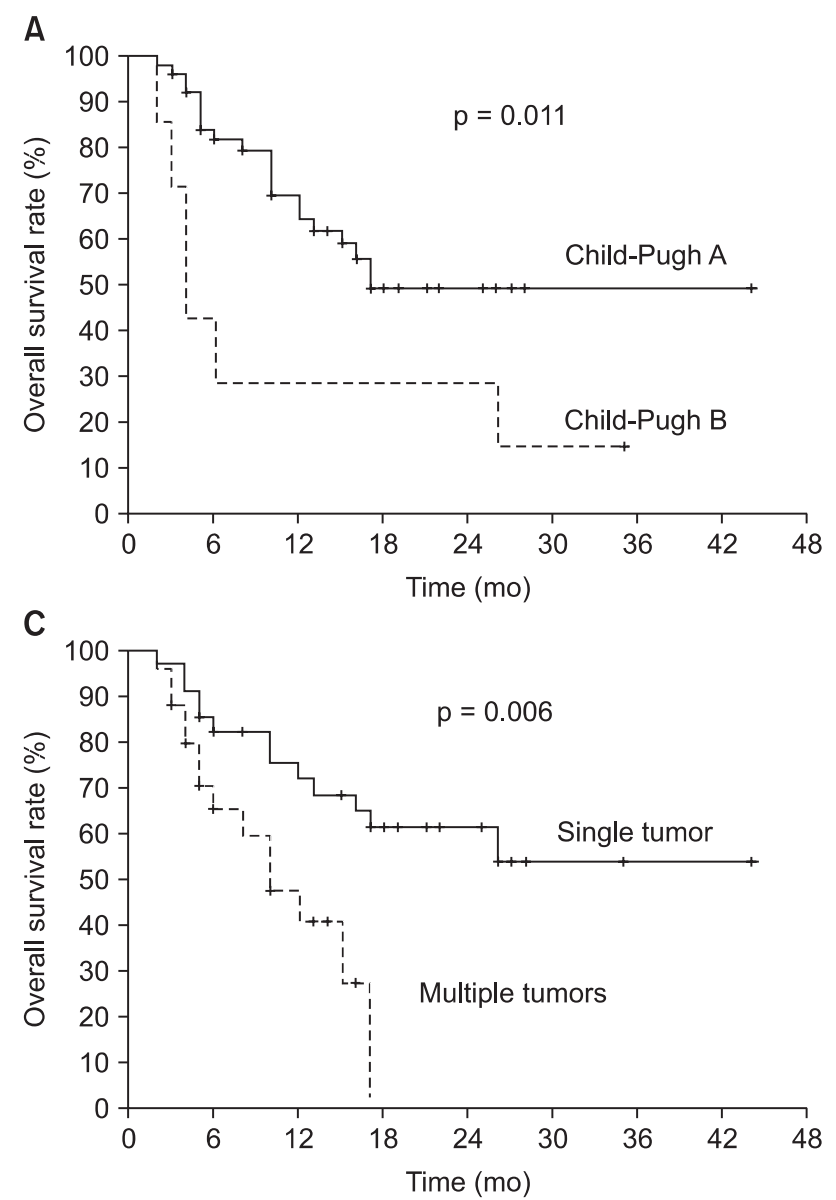

Fig. 3. Overall survival rate according to (A) Child-Pugh class, multiplicity, and (D) overall response to treatment. over TACE alone. For a primary tumor, RT can eliminate residual cancer cells at the tumor periphery that may remain viable due to blood supply from the collateral circulation or recanalization of the embolized artery. For PVTT, RT may help maintain liver function by stabilizing the patency of portal flow and consequently, enabling to perform additional TACE $[7,8]$. Additionally, RT may increase the efficacy of subsequent TACE by decreasing shunt flow around the PVTT [24]. Conversely, TACE can be helpful to control intrahepatic metastasis outside the RT field. In addition, it has a radio-sensitizing effect due to Adriamycin, which is injected through the tumor during TACE and maintained for up to 27 days [12]. Therefore, the addition of RT after TACE within 2 weeks can theoretically maximize the combining effect of two modalities if not the liver function deteriorates.

Although randomized trials are lacking, several studies have reported the efficacy of combined treatment with TACE and RT in patients with advanced HCC [7-9,14-18]. A response rate of
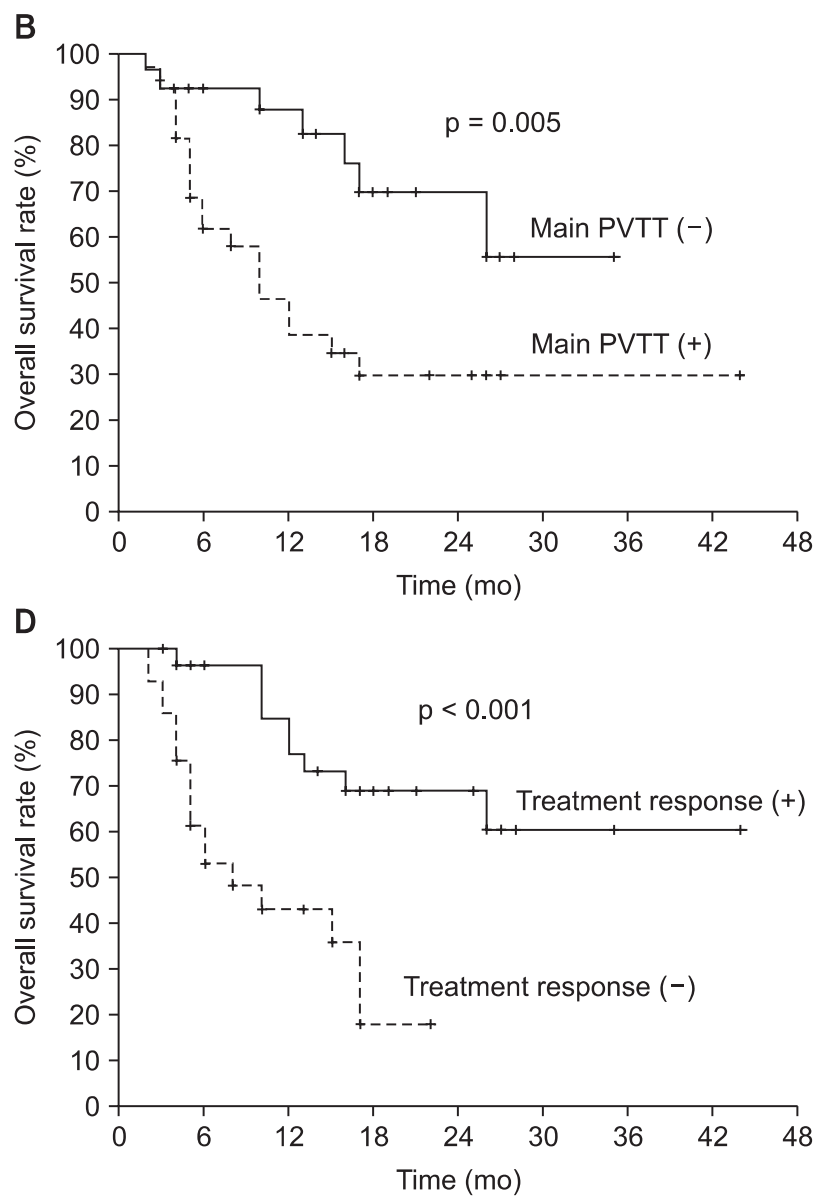

(B) the presence of main portal vein tumor thrombosis (PVTT), (C) http://dx.doi.org/10.3857/roj.2014.32.1.14

www.e-roj.org 19 
Table 2. Acute adverse events after TACE followed by RT

\begin{tabular}{|c|c|c|c|c|}
\hline \multirow{2}{*}{ Adverse event } & \multicolumn{2}{|c|}{ Events after TACE } & \multicolumn{2}{|c|}{ Events after RT } \\
\hline & Grade 1 or 2 & Grade 3 or higher & Grade 1 or 2 & Grade 3 or higher \\
\hline \multicolumn{5}{|l|}{ Symptoms } \\
\hline A/N/N & $24(40)$ & 0 & $28(47)$ & 0 \\
\hline Abdominal pain & $44(75)$ & 0 & 7 (12) & 0 \\
\hline Fever/chills & $15(25)$ & 0 & $5(8)$ & 0 \\
\hline Ascites & $2(3)$ & 0 & $6(10)$ & $2(3)$ \\
\hline \multicolumn{5}{|l|}{ Laboratory } \\
\hline AST & $18(30)$ & $40(68)$ & $39(66)$ & $2(3)$ \\
\hline ALT & $28(47)$ & $27(46)$ & $19(32)$ & $1(2)$ \\
\hline ALP & $35(59)$ & $2(3)$ & 31 (52) & 0 \\
\hline Bilirubin & $30(51)$ & $2(3)$ & $9(15)$ & $2(3)$ \\
\hline Albumin & $17(29)$ & 0 & $27(46)$ & 0 \\
\hline
\end{tabular}

Values are presented as number of patients (\%).

TACE, transcatheter arterial chemoembolization; RT, radiotherapy; A/N/N, anorexia/nausea/vomiting; AST, aspartate aminotransferase; ALT, alanine aminotransferase; ALP, alkaline phosphatase.

$25 \%$ to $76 \%$ and a median survival of 5.3 to 20 months have been reported. However, the results are difficult to interpret due to heterogeneity in the study population, the time interval between TACE and RT, and the history of prior treatment for HCC. In the current study, we selected treatment-naïve patients with HCC who were diagnosed as BCLC stage $C$ to ensure that we had a homogenous study population. We also excluded patients with extrahepatic spread because extrahepatic spread could not be encompassed within the RT portals together with the main tumor, and sorafenib is considered to be the first-line therapy for systemic disease. For these patients, we showed favorable outcomes when treated with TACE followed by RT with a 2-week interval. The overall response rate at 1 month was 51\% and the median OS was 17 months. Furthermore, the 3-year OS rate was around $50 \%$ in patients with favorable factors, such as Child-Pugh A or the absence of main PVTT or single tumor or responded tumor to treatment (Fig. 3). In contrast, the use of sorafenib alone showed a median OS of 10.7 months in a study of North American and European patients and 6.5 months in a study of Asian-Pacific patients $[3,4]$. In the subgroup analysis of AsianPacific study, even the patients with the best prognosis, who had no vascular invasion or extrahepatic spread, had a median survival of 14.3 months [25]. A recent study compared TACE alone with sorafenib for treatment of BCLC stage $C$ and found that TACE showed a similar survival outcome to sorafenib (TACE, 9.2 months; sorafenib, 7.4 months), even in the presence of vascular invasion or extrahepatic metastasis [6]. These findings suggest that an effective locoregional treatment can provide a survival benefit that is comparable or better than that of sorafenib in locally advanced HCC, although this subject will need to be validated in a prospective trial.

Previously, the major limitation of RT to the liver was the risk of hepatic toxicity. However, with recent technical advances, RT can be focused on the tumor while preserving the healthy liver. In the current study, 4D planning allowed the incorporation of patient-specific motion into the target volume, and imageguided RT may have facilitated more accurate radiation delivery and margin reduction. These efforts may make it more feasible to reduce RT-toxicity. Liver enzyme elevation of grade 3 or above was observed in only two patients (3\%); for these patients, RT was delivered with caution under our institution's guidelines. For GD toxicity, the severe toxicity (grade 3 ) that required major cauterization and transfusion occurred in only two patients (3\%). But the moderate toxicity (grade 2) that required the medication such as a proton pump inhibitor occurred in 14 patients (24\%). Liver cirrhosis and portal vein hypertension are the risk factors for GD ulcer [26-28], so caution should be used when the GD is in close proximity to the RT target volume. The factors that influence the development of GD toxicity are currently being investigated at our institution.

Our study has several limitations. We enrolled a relatively small number of patients, and we used a retrospective study design. Although we tried to overcome these limitations by enrolling a homogenous study population (i.e., treatmentnaïve $\mathrm{HCC}$ patients at $\mathrm{BCLC}$ stage $\mathrm{C}$ ), our results will need to be validated in a prospective study. In addition, we could not 
draw any conclusions regarding the efficacy of combined TACE and RT for patients with extrahepatic spread.

In conclusion, the combination treatment of TACE followed 2 weeks later by RT was safe and it showed favorable outcomes in treatment-naïve patients with locally advanced HCC. A prospective randomized trial is needed to validate these results.

\section{Conflict of Interest}

No potential conflict of interest relevant to this article was reported.

\section{Acknowledgments}

This research was supported by Basic Science Research Program through the National Research Foundation of Korea (NRF) funded by the Ministry of Education, Science and Technology (NRF-2012R1A1A2042414). This study was supported by Samsung Medical Center grant [GF01130081].

\section{References}

1. Bruix J, Sherman M; Practice Guidelines Committee, American Association for the Study of Liver Diseases. Management of hepatocellular carcinoma. Hepatology 2005;42:1208-36.

2. European Association for the Study of the Liver; European Organisation for Research and Treatment of Cancer. EASLEORTC clinical practice guidelines: management of hepatocellular carcinoma. J Hepatol 2012;56:908-43.

3. Cheng $A L$, Kang $Y K$, Chen $Z$, et al. Efficacy and safety of sorafenib in patients in the Asia-Pacific region with advanced hepatocellular carcinoma: a phase III randomised, doubleblind, placebo-controlled trial. Lancet Oncol 2009;10:25-34.

4. Llovet JM, Ricci S, Mazzaferro V, et al. Sorafenib in advanced hepatocellular carcinoma. N Engl J Med 2008;359:378-90.

5. Han KH, Kudo M, Ye SL, et al. Asian consensus workshop report: expert consensus guideline for the management of intermediate and advanced hepatocellular carcinoma in Asia. Oncology 2011;81 Suppl 1:158-64.

6. Pinter M, Hucke F, Graziadei I, et al. Advanced-stage hepatocellular carcinoma: transarterial chemoembolization versus sorafenib. Radiology 2012;263:590-9.

7. Zeng ZC, Fan J, Tang ZY, et al. A comparison of treatment combinations with and without radiotherapy for hepatocellular carcinoma with portal vein and/or inferior vena cava tumor thrombus. Int J Radiat Oncol Biol Phys 2005;61:432-43.

8. Yoon SM, Lim YS, Won HJ, et al. Radiotherapy plus transarterial chemoembolization for hepatocellular carcinoma invading the portal vein: long-term patient outcomes. Int J Radiat Oncol Biol Phys 2012;82:2004-11.

9. Xu LT, Zhou ZH, Lin JH, et al. Clinical study of transarterial chemoembolization combined with 3-dimensional conformal radiotherapy for hepatocellular carcinoma. Eur J Surg Oncol 2011;37:245-51.

10. Oh D, Lim DH, Park HC, et al. Early three-dimensional conformal radiotherapy for patients with unresectable hepatocellular carcinoma after incomplete transcatheter arterial chemoembolization: a prospective evaluation of efficacy and toxicity. Am J Clin Oncol 2010;33:370-5.

11. Li B, Yu J, Wang L, et al. Study of local three-dimensional conformal radiotherapy combined with transcatheter arterial chemoembolization for patients with stage III hepatocellular carcinoma. Am J Clin Oncol 2003;26: e92-9.

12. Nakamura $H$, Hashimoto $T, O i$, Sawada S. Transcatheter oily chemoembolization of hepatocellular carcinoma. Radiology 1989;170(3 Pt 1):783-6.

13. Yu Jl, Park HC, Lim DH, et al. Scheduled interval trans-catheter arterial chemoembolization followed by radiation therapy in patients with unresectable hepatocellular carcinoma. J Korean Med Sci 2012;27:736-43.

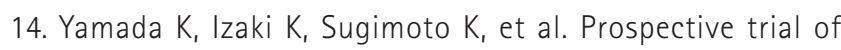
combined transcatheter arterial chemoembolization and three-dimensional conformal radiotherapy for portal vein tumor thrombus in patients with unresectable hepatocellular carcinoma. Int J Radiat Oncol Biol Phys 2003;57:113-9.

15. Park W, Lim DH, Paik SW, et al. Local radiotherapy for patients with unresectable hepatocellular carcinoma. Int J Radiat Oncol Biol Phys 2005;61:1143-50.

16. Meng MB, Cui $Y L, L u Y$, et al. Transcatheter arterial chemoembolization in combination with radiotherapy for unresectable hepatocellular carcinoma: a systematic review and metaanalysis. Radiother Oncol 2009;92:184-94.

17. Park SG, Kim JH, Byun SJ, et al. Radiation therapy for hepatocellular carcinoma with portal vein tumor thrombosis. J Korean Soc Ther Radiol Oncol 2011;29:36-43.

18. Kim JH, Choi EK, Ahn SD, et al. The role of radiotherapy in the treatment of portal vein thrombosis from advanced hepatocellular carcinoma. J Korean Soc Ther Radiol Oncol 2007;25:170-6.

19. Marrero JA, Kudo M, Bronowicki JP. The challenge of prognosis and staging for hepatocellular carcinoma. Oncologist 2010;15 Suppl 4:23-33.

20. Wang JH, Changchien CS, Hu TH, et al. The efficacy of treatment schedules according to Barcelona Clinic Liver Cancer staging for hepatocellular carcinoma: survival analysis of 3892 patients. Eur J Cancer 2008;44:1000-6.

21. Lim DH, Lee $H$, Park $H C$, et al. The efficacy of high-dose 
3-dimensional conformal radiation therapy in patients with small hepatocellular carcinoma not eligible for other local modalities. Am J Clin Oncol 2013;36:162-6.

22. Kudo $M$, Izumi $N$, Kokudo $N$, et al. Management of hepatocellular carcinoma in Japan: Consensus-Based Clinical Practice Guidelines proposed by the Japan Society of Hepatology (JSH) 2010 updated version. Dig Dis 2011;29:339-64.

23. Korean Liver Cancer Study Group; Korea National Cancer Center. Practice guidelines for management of hepatocellular carcinoma 2009. Korean J Hepatol 2009;15:391-423.

24. Hsu HC, Chen TY, Chiu KW, et al. Three-dimensional conformal radiotherapy for the treatment of arteriovenous shunting in patients with hepatocellular carcinoma. Br J Radiol 2007;80: 38-42.

25. Cheng AL, Guan $Z$, Chen $Z$, et al. Efficacy and safety of sorafenib in patients with advanced hepatocellular carcinoma according to baseline status: subset analyses of the phase III Sorafenib Asia-Pacific trial. Eur J Cancer 2012;48:1452-65.

26. Tomoda J, Mizuno M, Sugihara T, Itano T, Tsuji T. Gastric mucosal lesion in liver disease: impaired gastric mucosal defence mechanism in rats with induced liver injury and in patients with liver cirrhosis. J Gastroenterol Hepatol 1989;4 Suppl 1:136-9.

27. Chen LS, Lin HC, Hwang SJ, Lee FY, Hou MC, Lee SD. Prevalence of gastric ulcer in cirrhotic patients and its relation to portal hypertension. J Gastroenterol Hepatol 1996;11:59-64.

28. Chon YE, Seong J, Kim BK, et al. Gastroduodenal complications after concurrent chemoradiation therapy in patients with hepatocellular carcinoma: endoscopic findings and risk factors. Int J Radiat Oncol Biol Phys 2011;81:1343-51. 